\title{
Hypercholesterolaemia increases the risk of high-turnover osteoporosis in men
}

\author{
YANMAN ZHOU ${ }^{1-3}$, TUO DENG $^{1-3}$, HAIQING ZHANG ${ }^{1-3}$, QINGBO GUAN ${ }^{1-3}$, HONGQIANG ZHAO $^{4}$, \\ CHUNXIAO YU ${ }^{1-3}$, SHANSHAN SHAO ${ }^{1-3}$, MENG ZHAO $^{1-3}$ and JIN XU ${ }^{1-3}$ \\ ${ }^{1}$ Department of Endocrinology, Shandong Provincial Hospital Affiliated to Shandong University; \\ ${ }^{2}$ Shandong Key Laboratory of Endocrinology and Lipid Metabolism; ${ }^{3}$ Institute of Endocrinology and Metabolism, \\ Shandong Academy of Clinical Medicine, Jinan, Shandong 250021; ${ }^{4}$ Department of Internal Medicine, \\ Laiwu People's Hospital, Laiwu, Shandong 271100, P.R. China
}

Received July 26, 2018; Accepted March 8, 2019

DOI: $10.3892 / \mathrm{mmr} .2019 .10131$

\begin{abstract}
As the incidence of osteoporosis (OP) and hypercholesterolaemia in men has increased, male OP has drawn more attention from clinicians worldwide. The present study sought to investigate the effects of cholesterol on male bone. Between July 2015 and October 2015, 216 men (aged $\geq 18$ years) were recruited for this cross-sectional study. To test our clinical hypothesis, we designed two male animal models: Exogenous hypercholesterolaemia induced by a high-cholesterol diet (HCD) and endogenous hypercholesterolaemia induced by apolipoprotein E (ApoE) knockout. Finally, the direct effects of cholesterol on osteoblasts were observed in cell experiments. In our clinical studies, men with hypercholesterolaemia displayed a lower bone mineral density (BMD) and increased beta collagen cross-linking (beta-CTX) and type I anterior collagen amino terminal peptide (PINP) levels compared to those of the control subjects. Serum cholesterol levels were a significant independent predictor of BMD, beta-CTX and PINP and were negatively correlated with BMD and positively correlated with beta-CTX and PINP levels. Our animal experimental results validated our clinical results, as they also indicated that hypercholesterolaemia damages bone microstructure and reduces bone strength. Cholesterol directly increased osteoblast functional gene expression in vitro. Hypercholesterolaemia increases the risk of high-turnover osteoporosis in men at least in part by excessively promoting the activity of the remodelling pathway. In addition, hypercholesterolaemia damages the bone microstructure, resulting in osteopenia or OP and reduced bone strength, leading to a higher risk of fracture in men. We emphasize the importance
\end{abstract}

Correspondence to: Dr Jin Xu, Department of Endocrinology, Shandong Provincial Hospital Affiliated to Shandong University, 324 Jing 5 Road, Jinan, Shandong 250021, P.R. China

E-mail: xujin267903@163.com

Key words: hypercholesterolaemia, osteoporosis, high cholesterol diet, $\mathrm{ApoE}^{-/-}$rat, osteoblast of preventing and treating hypercholesterolaemia as well as monitoring bone metabolic markers and BMD in men with hypercholesterolemia for the effective prevention of bone loss and subsequent fracture. In addition, our findings provide a theoretical basis for the development of treatments for high cholesterol-induced osteoporosis in men.

\section{Introduction}

Osteoporosis (OP) can be diagnosed indirectly via non-invasive measurements of bone mineral density (BMD) (1). In recent years, men have been shown to be affected by this 'silent epidemic'. The prevalence of OP was found to be between 6 and $22 \%$ in men (2). The lifetime risk of osteoporotic fractures in men is approximately one-third of that in women (3). OP-related fractures are associated with higher mortality rates in men than in women (4). Thus, male OP should draw more attention from clinicians worldwide.

The relationship between hypercholesterolemia and osteoporosis in men has been preliminarily discovered (5). To the best of our knowledge, the widespread and profound effects of hypercholesterolaemia on male bone and their underlying mechanisms have seldom been reported thus far. As the prevalence of hypercholesterolaemia in male adults is increasing, and is even higher than that in females (6), the effects of hypercholesterolaemia on male bone should attract sufficient attention. Therefore, we aimed to investigate the effects of hypercholesterolaemia on male bone and the underlying mechanisms.

\section{Materials and methods}

Human subjects and design. Between July 2015 and October 2015, 216 men (aged $\geq 18$ years) were recruited by the Jinan Health Organization (Jinan, China). All subjects completed a self-reported questionnaire including items pertaining to their demographic characteristics, lifestyle habits, dietary habits, medical histories and current medications. Height and body weight were measured to calculate body mass index (BMI) using the following formula: BMI=body weight $(\mathrm{kg}) /$ height $\left(\mathrm{m}^{2}\right)$. Fasting blood samples were drawn for clinical laboratory tests. 
The exclusion criteria included participants with chronic diseases, hepatic, renal, thyroid, other endocrine diseases, diabetes and hypertriglyceridemia and participants taking medications such as glucocorticoids, testosterone, thyroid hormone, bisphosphonates, calcitonin, calcium and active vitamin D analogues. Overall, 216 men were enrolled in the present study, which was approved by the Ethics Committee of Provincial Hospital Affiliated with Shandong University (Jinan, China) and all participants provided written informed consent before participating in the study.

According to the criteria outlined in China's Adult Dyslipidemia Prevention Guide (2016 version) (7), the 216 men enrolled in the present study were divided into a hypercholesterolaemia group (total cholesterol $\geq 5.20 \mathrm{mmol} / \mathrm{l}, \mathrm{n}=102$ ) and a control group (total cholesterol $<5.20 \mathrm{mmol} / \mathrm{l}, \mathrm{n}=114$ ).

Clinical laboratory tests and BMD measurements. Serum total cholesterol (TC), triglyceride (TG), low-density lipoprotein cholesterol (LDL-C), high-density lipoprotein cholesterol (HDL-C) and fasting plasma glucose (FPG) levels were measured by the Beckman Chemistry Analyzer AU5800 System 195 (Beckman Coulter, Inc., Tokyo, Japan) and beta collagen cross-linking (beta-CTX), type I anterior collagen amino terminal peptide (PINP), testosterone (To), free thyroxine (FT4), thyroid stimulating hormone (TSH) and 25-hydroxyvitamin D [25(OH)D] concentrations were determined by an electrochemiluminescence immunoassay (Cobas 8000 analyser; Roche Diagnostics, Basel, Switzerland) at the Clinical Laboratory of Shandong Provincial Hospital. BMD was measured in the low-median region of the left forearm by a trained technologist using a dual-energy X-ray absorptiometry (DXA) fan beam bone densitometer (EXA-3000; OsteoSys, Co., Ltd., Seoul, Korea).

Animal experimental design. The animal experimental protocol was approved by the Animal Ethics Committee of Shandong Provincial Hospital. Two animal models were used in this study. For the first model, 24 6-week-old male Sprague-Dawley rats weighing approximately $200 \mathrm{~g}$ were obtained from Vital River Laboratory Animal Technology Co, Ltd. (Beijing, China). Following being acclimated to their housing environments for 1 week, the rats received one of two diets, a normal control diet (NCD, 100\% standard rodent chow) or a high cholesterol diet (HCD, $2 \%$ cholesterol $+98 \%$ standard rodent chow), and were randomly assigned into three groups: An NCD group (36 weeks), an HCD group (36 weeks), or an HCD+NCD group (24 weeks HCD+12 weeks NCD). Rats of the second model included male wild-type $\left(\mathrm{ApoE}^{+/+}\right)$rats and male ApoE-knockout $(\mathrm{KO})$ $\left(\mathrm{ApoE}^{-/}\right)$rats, which were obtained by breeding heterozygous $\left(\mathrm{ApoE}^{+/}\right)$rats purchased from Beijing Biocytogen Co., Ltd. (Beijing, China) and were fed an NCD for 20 weeks. All animals were maintained in temperature-controlled rooms $\left(22-25^{\circ} \mathrm{C}\right)$ under 12-h light/dark cycles and allowed free access to food and water. All rats were sacrificed by exsanguination following anesthesia with subcutaneous injection of $30 \mathrm{mg} / \mathrm{kg}$ pentobarbital sodium at the end of the feeding period after being fasted for $8 \mathrm{~h}$. Body weights were obtained, and fasting blood samples were withdrawn. The left femurs were collected and cleaned of soft tissue before being packed in gauze soaked with a $\mathrm{NaCl}$ solution $(9 \mathrm{~g} / \mathrm{l})$ and stored at $-30^{\circ} \mathrm{C}$.
Serum parameter analysis. Serum lipid profiles were measured by an Olympus AU5400 automatic biochemical analyser (Olympus Corp., Mishama, Japan). Serum tartrate-resistant acid phosphatase (TRAP), beta-CTX, PINP, bone alkaline phosphatase (ALPL) and bone glaprotein (BGP) were measured with ELISA kits (Uscn Life Science, Inc., Wuhan, China).

Micro-CT (mCT) measurements. The trabecular and cortical microarchitectures of the left femurs were analysed using mCT (SkyScan 1176; Bruker-microCT, Kontich, Belgium). The slice thickness was $17.93 \mu \mathrm{m}$, and the voxel resolution was $22 \mu \mathrm{m}^{3}$. Quantitative analyses of the morphometric parameters were conducted using the appropriate software package, and 3D images of the distal femur and femoral diaphysis were reconstructed for visualization. All analyses were conducted by a trained professional in a blinded manner who was unaware of the treatments to which the specimens had been subjected.

Biomechanical property measurement. We assessed the mechanical properties of the femoral diaphysis as previously reported (8) using an ElectroForce 3230 Bose System (Bose, Minnetonka, MN, USA). Based on these parameters reported by the instrument, we analysed the mechanical properties of the femoral diaphysis.

Primary culture of osteoblasts. Primary osteoblasts obtained from new-born rats were cultured as described in a previous study (9). The cells were cultured in 6-well plates at a density of $1 \times 10^{5}$ cells/well in Dulbecco's modified Eagle's medium (DMEM; Gibco; Thermo Fisher Scientific, Inc., Waltham, MA, USA) containing $10 \%$ (v/v) fetal bovine serum (FBS; Biochrom $\mathrm{GmbH}$, Berlin, Germany), $100 \mathrm{U} / \mathrm{ml}$ penicillin-streptomycin solution (Sigma-Aldrich; Merck KGaA, Darmstadt, Germany) and $2 \mathrm{mM}$ L-glutamine (Sigma-Aldrich; Merck KGaA) at $37^{\circ} \mathrm{C}$ in a humidified $5 \% \mathrm{CO}_{2}$ atmosphere. The medium was refreshed every $48 \mathrm{~h}$. Haematoxylin and eosin (H\&E) staining was used to identify the typical morphological characteristics of the osteoblasts. Alkaline phosphatase (ALP) (Genmed, Shanghai, China) staining, Alizarin red (Beijing Solarbio Science \& Technology Co. Ltd. (Beijing, China) staining and Von Kossa (Genmed) staining were performed for osteoblast phenotypic and functional identification. After 3 days of incubation at $37^{\circ} \mathrm{C}$ in a humidified $5 \% \mathrm{CO}_{2}$ atmosphere, the primary osteoblasts were starved of serum for $2 \mathrm{~h}$ and then treated with cholesterols (Sigma-Aldrich; Merck KGaA; 0,10 and $20 \mu \mathrm{g} / \mathrm{ml}$ ) for 12,24 and $48 \mathrm{~h}$. We subsequently analysed osteoblast function by measuring gene expression.

Reverse transcription-quantitative polymerase chain reaction (RT-qPCR). Total RNA was extracted using TRIzol (Takara Biotechnology Co., Ltd., Dalian, China) according to the manufacturer's instructions. cDNA was synthesized from 500 ng of total RNA using ReverTra Ace reverse transcriptase (Takara Biotechnology Co., Ltd.) and oligo dT primers (Takara Biotechnology Co., Ltd.). The following PCR primers were used in the study: ALPL: Forward (F), 5'-CATCGCCTA TCAGCTAATGCACA-3' and reverse (R), 5'-ATGAGGTCC AGGCCATCCAG-3'; collagen I: F, 5'-GACATGTTCAGC TTTGTGGACCTC-3' and R, 5'-AGGGACCCTTAGGCC 
Table I. Clinical characteristics of the subjects with hypercholesterolemia and control subjects.

\begin{tabular}{lccr}
\hline Characteristics $($ mean \pm SD) & Controls $(\mathrm{n}=112)$ & Hypercholesterolemia $(\mathrm{n}=104)$ & Total $(\mathrm{n}=216)$ \\
\hline Age $(\mathrm{years})$ & $48.140 \pm 13.750$ & $50.098 \pm 13.995$ & $49.065 \pm 13.868$ \\
BMI $\left(\mathrm{kg} / \mathrm{m}^{2}\right)$ & $25.337 \pm 3.810$ & $25.256 \pm 3.031$ & $25.299 \pm 3.456$ \\
FPG $(\mathrm{mmol} / \mathrm{l})$ & $5.421 \pm 0.619$ & $5.462 \pm 0.469$ & $5.462 \pm 0.562$ \\
HbA1 $(\%)$ & $5.503 \pm 1.139$ & $5.433 \pm 0.342$ & $5.454 \pm 0.365$ \\
To $(\mathrm{ng} / \mathrm{ml})$ & $5.652 \pm 1.624$ & $5.669 \pm 2.266$ & $5.497 \pm 1.972$ \\
FT4 $(\mathrm{pmol} / \mathrm{l})$ & $19.256 \pm 2.143$ & $18.913 \pm 2.704$ & $19.094 \pm 2.425$ \\
TSH $(\mu \mathrm{IU} / \mathrm{ml})$ & $2.338 \pm 1.691$ & $2.465 \pm 1.555$ & $2.398 \pm 1.626$ \\
25(OH)D $(\mathrm{ng} / \mathrm{ml})$ & $28.448 \pm 9.183$ & $28.257 \pm 10.048$ & $28.358 \pm 9.579$ \\
TG $(\mathrm{mmol} / \mathrm{l})$ & $1.201 \pm 0.328$ & $1.27 \pm 0.290$ & $1.234 \pm 0.312$ \\
TC $(\mathrm{mmol} / \mathrm{l})$ & $4.485 \pm 0.496$ & $5.737 \pm 0.412^{\mathrm{b}}$ & $5.076 \pm 0.776$ \\
LDL-C $(\mathrm{mmol} / \mathrm{l}$ & $2.789 \pm 0.406$ & $3.722 \pm 0.351^{\mathrm{b}}$ & $3.230 \pm 0.602$ \\
HLD-C $(\mathrm{mmol} / \mathrm{l}$ & $1.175 \pm 0.235$ & $1.309 \pm 0.262^{\mathrm{b}}$ & $1.238 \pm 0.257$ \\
BMD $\left.(\mathrm{g} / \mathrm{cm})^{2}\right)$ & $0.536 \pm 0.066$ & $0.505 \pm 0.092^{\mathrm{b}}$ & $0.522 \pm 0.081$ \\
Beta-CTX $(\mathrm{ng} / \mathrm{ml})$ & $0.437 \pm 0.164$ & $0.5 \pm 0.201^{\mathrm{a}}$ & $0.467 \pm 0.185$ \\
PINP $(\mathrm{ng} / \mathrm{ml})$ & $46.974 \pm 16.259$ & $52.477 \pm 19.011^{\mathrm{a}}$ & $49.572 \pm 17.785$ \\
\end{tabular}

BMI, body mass index; FPG, fasting plasma glucose; HbA1c, haemoglobin A1c; To, testosterone; FT4, free thyroxine; TSH, thyroid stimulating hormone; 25(OH)D, 25-hydroxyvitamin D; TG, triglyceride; TC, total cholesterol; LDL-C, low-density lipoprotein cholesterol; HDL-C, high-density lipoprotein cholesterol; BMD, bone mineral density; beta-CTX, beta collagen cross-linking; PINP, type I anterior collagen amino terminal peptide. ${ }^{\mathrm{a}} \mathrm{P}<0.05,{ }^{\mathrm{b}} \mathrm{P}<0.01$, significant results.

ATTGTGTA-3'; BGP: F, 5'-GGACCCTCTCTCTGCTCA CTCT-3' and R, 5'-CTTACTGCCCTCTGCTTGG-3'; $\beta$-actin: F, 5'-ACCCAGATCATGTTTGAGAC-3' and R, 5'-GTCAGG ATCTTCATGAGGTAGT-3'.

Extracellular protein ELISA. Secreted BGP and ALPL levels were measured with ELISA kits (Uscn Life Science, Inc.) according to the manufacturers' protocols. The protein concentration of the cell homogenate was determined using a BCA assay (Shen Neng Bo Cai Corp., Shanghai, China). The levels of BGP and ALPL in the media were normalized to the total cellular protein content.

Statistical analysis. Statistical analyses were performed using SPSS 22 (IBM Corp., Armonk, NY, USA). All data are expressed as the mean \pm standard deviation for continuous variables. Unpaired Student's t-test or one-way analysis of variance (ANOVA) and LDS, S-N-K, Duncan and Dunnet post hoc tests were used to compare BMD, beta-CT X and PINP levels between hypercholesterolaemia and controls and all animal and cell measurement data. Multivariate linear regression analysis was used to evaluate the correlations between clinical parameters and serum BMD and beta-CTX and PINP levels. P-values $<0.05$ were considered to indicate a statistically significant result.

\section{Results}

Correlation between serum cholesterol and BMD, beta-CTX and PINP. Table I presents the characteristics of both groups. BMD was significantly lower in men with hypercholesterolaemia than in control subjects $(\mathrm{P}<0.001)$, and both beta-CTX and PINP levels were increased in the hypercholesterolaemia group compared with those in the controls $(\mathrm{P}<0.05)$. Moreover, serum TC, LDL-C and HDL-C levels were significantly higher among men with hypercholesterolaemia $(\mathrm{P}<0.001)$. There were no significant differences in the age, BMI or FPG, HbAc1, To, FT4, TSH, 25(OH)D and TG levels between the two groups.

To further study the effects of cholesterol on BMD and beta-CTX and PINP levels, we performed multiple linear regression analysis (Table II) in which beta-CTX and PINP served as dependent variables and other factors served as independent variables. As shown in Table II, TC was a significant independent predictor of BMD, beta-CTX and PINP and was negatively correlated with BMD and positively correlated with beta-CTX and PINP. LDL-C was positively correlated with beta-CTX levels but was not significantly correlated with BMD or PINP levels.

Effects of HCD and HCD withdrawal on bone in male rats. Serum cholesterol levels were higher in HCD rats than levels in the controls $(\mathrm{P}<0.01)$, inducing exogenous hypercholesterolaemia in these rats, and these levels were diminished in the HCD+NCD rats compared with those in the HCD rats $(\mathrm{P}<0.01)$, and there was no significant difference in $\mathrm{TG}$ or body weight among the three groups (Fig. 1A).

The bone mCT measurements are shown in Table III. HCD administration decreased trabecular and cortical vBMD (Fig. 1D) and caused microstructural damage (Fig. 1B and C and Table III); Fig. 1B is two-dimensional and cross-sectional scanning structure, and Fig. $1 \mathrm{C}$ is three-dimensional structure synthesized by computer analysis, showing the microstructure of bone in a more detailed manner. vBMD was higher in the $\mathrm{HCD}+\mathrm{NCD}$ rats than that in the HCD male rats (Fig. 1D). The 
Table II. Multiple regression analysis between BMD, serum Beta-CTX or PINP and TC, LDL-C, HDL-C.

\begin{tabular}{lccc}
\hline Characteristics & BMD & Beta-CTX & PINP \\
\hline Age & $-0.389^{\mathrm{b}}$ & $-0.241^{\mathrm{b}}$ & $-0.257^{\mathrm{b}}$ \\
BMI & $0.195^{\mathrm{b}}$ & -0.051 & -0.102 \\
To & $0.153^{\mathrm{a}}$ & -0.086 & 0.017 \\
TG & -0.024 & 0.049 & 0.123 \\
TC & $-0.175^{\mathrm{b}}$ & $0.169^{\mathrm{a}}$ & $0.166^{\mathrm{a}}$ \\
LDL-C & -0.073 & $0.172^{\mathrm{a}}$ & -0.068 \\
HLD-C & 0.073 & -0.038 & -0.021 \\
\hline
\end{tabular}

BMD, bone mineral density; beta-CTX, beta collagen cross-linking; PINP, type I anterior collagen amino terminal peptide; BMI, body mass index; To, testosterone; TG, triglyceride; TC, total cholesterol LDL-C, low-density lipoprotein cholesterol; HDL-C, high-density lipoprotein cholesterol. ${ }^{\mathrm{a}} \mathrm{P}<0.05,{ }^{\mathrm{b}} \mathrm{P}<0.01$, significant results.

levels of mCT 3D microstructural damage were ameliorated in HCD withdrawal rats compared with those in the HCD rats (Table III). These results suggest that HCD passively affected both trabecular and cortical bone, especially trabecular bone and that dietary modification can weaken these effects.

The levels of bone biomechanical parameters were increased significantly or tended to increase in HCD withdrawal rats compared with those in HCD rats (Fig. 1E and F). These results suggest that HCD caused deteriorations in bone strength and that dietary modification can mitigate these effects.

Our assessment revealed that the levels of serum bone resorption markers (TRAP and CTX-I) were significantly increased in HCD rats compared with those in the controls; however, they decreased after HCD withdrawal (Fig. 1G). Therefore, bone formation markers (BGP, ALPL and PINP) were identified (Fig. 1H). These results suggest that HCD administration elevated both bone resorption and bone formation, and dietary modification can alleviate these effects.

ApoE $^{-/-}$male rat bone loss. Serum cholesterol levels were significantly higher in $\mathrm{ApoE}^{-/-}$male rats than in $\mathrm{ApoE}^{+/+}$rats $(\mathrm{P}<0.01)$, inducing endogenous hypercholesterolaemia. There were no differences in TG levels or body weight between the two groups (Fig. 2A).

The levels of bone mCT measurements are shown in Table III. ApoE ${ }^{-/}$male rats displayed decreased trabecular and cortical vBMD (Fig. 2D) and microstructural damage (Fig. 2B and C) compared with $\mathrm{ApoE}^{+/ /}$rats. These results suggest that $\mathrm{ApoE}^{-/}$rats experienced significant trabecular and cortical bone loss and damage.

Regarding the biomechanical parameter measurements, the maximum load, maximum fracture load, stiffness and elastic modulus of the femoral diaphysis were apparently lower and energy absorption tended to decrease in $\mathrm{ApoE}^{-/}$rats compared with those in $\mathrm{ApoE}^{+/+}$rats (Fig. 2E and F).

Our assessment revealed that the serum levels of both bone resorption markers (TRAP and CTX-I) and bone formation markers (BGP, ALPL and PINP) were increased in $\mathrm{ApoE}^{-/-}$male rats compared with those in $\mathrm{ApoE}^{+/+}$rats (Fig. 2G and $\mathrm{H}$ ). These results suggest that $\mathrm{ApoE}^{-/}$rats had elevated bone resorption and bone formation, improving the bone turnover rate.

Effects of cholesterol on primary osteoblast function. We examined H\&E-stained osteoblasts via microscopy (Fig. 3A-a). These osteoblasts displayed cytoplasm that appeared violet in response to ALP staining (Fig. 3A-b). The cells were subjected to Alizarin red (Fig. 3A-c) or Von Kossa staining (Fig. 3A-d), revealing the presence of many round or elliptical opaque red or black nodules, which served as additional evidence indicating osteoblast mineralization. All of the above findings suggest that cultured rat calvaria osteoblasts are ideal experimental osteoblast model cells.

With increasing cholesterol doses, BGP, ALPL and collagen I mRNA expression as well as BGP and ALPL protein levels gradually increased compared with those in the control group (Fig. 3B and D) after treatment for $24 \mathrm{~h}$, but there were no differences in their levels among the different times tested $(12,24$ and $48 \mathrm{~h})$ after $10 \mu \mathrm{g} / \mathrm{ml}$ cholesterol treatment (Fig. 3C and E). These results suggested that cholesterol could increase the mRNA and protein expression levels of osteoblast functional genes in a dose-dependent but non-time-dependent manner.

\section{Discussion}

Currently, there is a dearth of information in the literature regarding the effects of hypercholesterolaemia on male bone metabolism. However, male osteoporosis (OP) should draw more attention from clinicians worldwide. Elucidating the effects of hypercholesterolaemia on male bone metabolism may aid in the search for answers regarding the relationship between hypercholesterolaemia and male OP and the underlying mechanisms and will further our understanding of the pathomechanisms underlying the relationship between the two diseases to reduce the financial burden imposed by male OP and OP-related hip fractures.

T2 diabetes mellitus (DM), low testosterone (To) and obesity are often associated with elevated plasma cholesterol levels (10-12). Diabetes mellitus (both type 1 and type 2) (13), testosterone deficiency $(14,15)$, hyperthyroidism and hypothyroidism (16), body mass index (BMI) (17), and hypertriglyceridemia $(18,19)$ reportedly affect bone mineral density (BMD). In this clinical study, we excluded participants with thyroid diseases, diabetes and hypertriglyceridemia according to clinical laboratory test results. There were no differences in BMI or serum fasting plasma glucose (FPG), $\mathrm{HbAc1}$, To, FT4, thyroid stimulating hormone (TSH), 25(OH)D and triglyceride (TG) levels between hypercholesterolaemia and control subjects. Therefore, we investigated the effects of hypercholesterolaemia on male bone, excluding the effects of the abovementioned parameters. Multiple regression analysis suggested that cholesterol was a significant independent predictor of BMD and negatively correlated with BMD. Some clinical studies have demonstrated that cholesterol-lowering statin drugs increase BMD and reduce the risk of OP-associated fractures $(20,21)$, supporting our results. We also found that serum cholesterol was a significant independent predictor 
A a

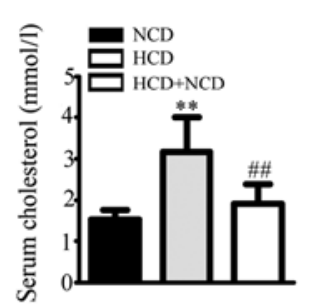

b

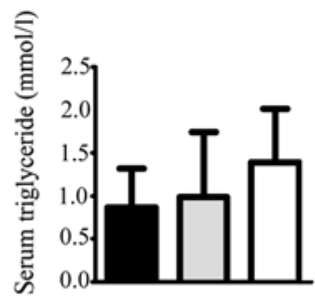

c

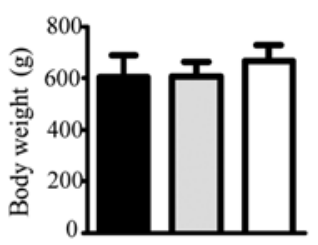

B

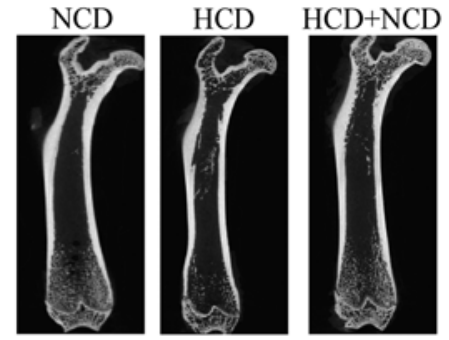

C

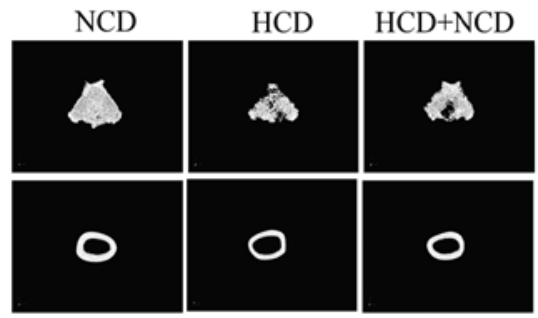

E a

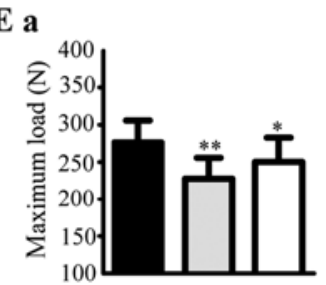

G a

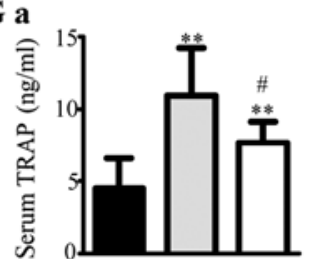

b そ
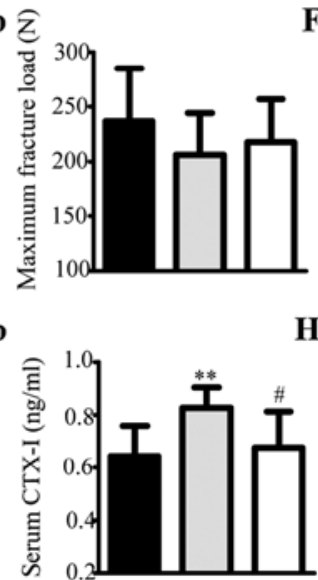

F a

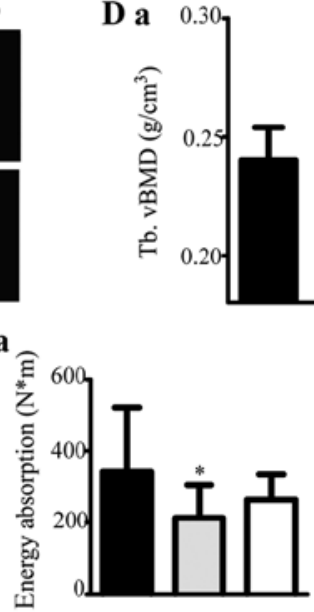

D a b

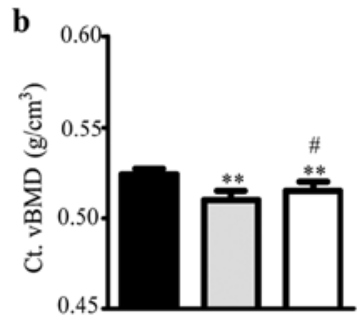

b

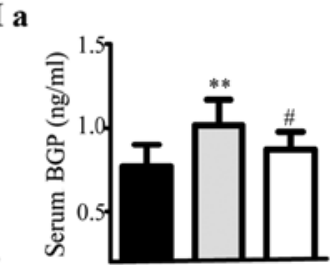

c

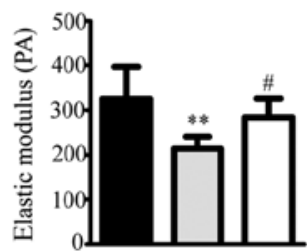

b

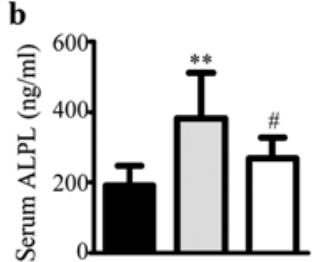

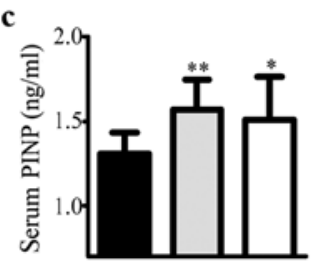

Figure 1. Effects of exogenous hypercholesterolaemia induced by HCD administration on male rats. (A) Serum (a) TC and (b) TG levels, and (c) body weight of the rats. (B) Representative 2D mCT images showing the trabecular and cortical microarchitectures of the femurs of each group. (C) Representative 3D mCT images showing the trabecular microarchitecture of the distal femur and the cortical microarchitecture of the midfemoral diaphyses. (D) The trabecular volumetric BMD (Tb. vBMD) (a) and cortical volumetric BMD (Ct. vBMD) (b) of the femur, as analysed via mCT. (E and F) The bone biomechanical parameters of the femoral diaphysis were calculated from a three-point bending test, and the maximum load (E-a), maximum fracture load (E-b), energy absorption (F-a), stiffness (F-b) and elastic modulus (F-c) of the femoral diaphysis were analysed. (G) The levels of the serum bone resorption markers TRAP (a) and beta-CTX (b) in rats were measured using ELISA kits. (H) The levels of serum bone formation markers BGP (a), ALPL (b) and PINP (c) in rats were measured using ELISA kits. ${ }^{\mathrm{P}}<0.05$ and ${ }^{* *} \mathrm{P}<0.01$ vs. the corresponding NCD group, ${ }^{\#} \mathrm{P}<0.05$ and ${ }^{\# /} \mathrm{P}<0.01$ vs. the corresponding HCD group. HCD, high cholesterol diet; TC, total cholesterol; LDL-C, low-density lipoprotein cholesterol; HDL-C, high-density lipoprotein cholesterol; TG, triglyceride; BMD, bone mineral density; BGP, bone glaprotein; ALPL, alkaline phosphatase; PINP, type I anterior collagen amino terminal peptide; NCD, normal control diet.

of beta-CTX and PINP levels and positively correlated with both. Beta-CTX fragments are released into the blood after type I collagen is dissolved by osteoclasts and are recognized as clinical indices of osteoclast activity and bone resorption. PINP directly reflects the rate of osteoblast-mediated collagen synthesis and is recognized as a clinical index of osteoblast activity and bone formation. Thus, we hypothesized that hypercholesterolaemia can promote the development of male OP by promoting both osteoclast-mediated bone resorption and osteoblast-mediated osteogenesis. Our data were inconsistent with those of a previous study (22). Majima et al (22) noted that higher TC levels were associated with higher bone turnover marker levels in female patients but not in male patients, perhaps because their study did not exclude other factors, such as male gonadal hypofunction, as stated by the authors in the indicated report. Importantly, the authors did not analyze the association between cholesterol and BMD. We used multiple regression analysis to exclude the effects of FPG, HbAc1, To, FT4, TSH, 25(OH)D and TG on bone, and we believe that our findings are more convincing.

Bone microstructural damage results in low BMD or $\mathrm{OP}$, and reduced bone strength is correlated with a higher risk of bone fracture. Thus, to test our clinical discovery and further explore the effects of hypercholesterolaemia on male bone microstructure and bone strength, we designed two male animal models. High cholesterol diet (HCD)-induced exogenous hypercholesterolaemia and ApoE-knockout (KO)-induced endogenous hypercholesterolaemia were observed in male rats.

HCD administration significantly increased serum cholesterol levels but not serum TG levels or body weight. Halade et al (23) reported that high fat diet-induced hypertriglyceridemia promotes bone marrow adiposity with a lower bone mass. Tatsumi et al (24) reported that higher weights 
Table III. Micro-CT measurements in male rats.

\begin{tabular}{|c|c|c|c|c|c|}
\hline Parameters (abbreviations) & NCD & HCD & $\mathrm{HCD}+\mathrm{NCD}$ & WT & $\mathrm{ApoE}^{-/-}$ \\
\hline \multicolumn{6}{|l|}{ Trebecular parameter } \\
\hline Percent bone volume (\%) (BV/TV) & $32.615 \pm 7.401$ & $18.01 \pm 3.901^{\mathrm{b}}$ & $25.614 \pm 2.834^{\mathrm{a}, \mathrm{d}}$ & $42.858 \pm 5.627$ & $33.137 \pm 4.434^{\mathrm{b}}$ \\
\hline Bone surface density $(1 / \mathrm{mm})(\mathrm{BS} / \mathrm{TV})$ & $8.533 \pm 1.592$ & $5.687 \pm 1.138^{\mathrm{b}}$ & $6.05 \pm 0.907^{\mathrm{b}}$ & $12.359 \pm 0.941$ & $10.329 \pm 0.711^{\mathrm{b}}$ \\
\hline Trabecular thickness (mm) (Tb.Th) & $0.142 \pm 0.01$ & $0.131 \pm 0.008^{\mathrm{b}}$ & $0.131 \pm 0.003^{\mathrm{a}}$ & $0.125 \pm 0.006$ & $0.112 \pm 0.004^{\mathrm{b}}$ \\
\hline Trabecular number $(1 / \mathrm{mm})(\mathrm{Tb} . \mathrm{N})$ & $2.398 \pm 0.492$ & $1.45 \pm 0.373^{\mathrm{b}}$ & $1.743 \pm 0.248^{\mathrm{b}}$ & $3.461 \pm 0.334$ & $2.916 \pm 0.332^{\mathrm{b}}$ \\
\hline Connectivity density $\left(1 / \mathrm{mm}^{3}\right)$ (Conn.Dn) & $57.15 \pm 15.627$ & $24.165 \pm 6.569^{b}$ & $28.796 \pm 8.819^{b}$ & $94.164 \pm 8.384$ & $110.377 \pm 17.264^{2}$ \\
\hline Trabecular separation (mm) (Tb.Sp) & $0.307 \pm 0.067$ & $0.542 \pm 0.116^{\mathrm{b}}$ & $0.452 \pm 0.105^{\mathrm{c}}$ & $0.210 \pm 0.028$ & $0.278 \pm 0.051^{\mathrm{b}}$ \\
\hline Trabecular pattern factor $(1 / \mathrm{mm})(\mathrm{Tb} . \mathrm{Pf})$ & $2.66 \pm 1.841$ & $7.721 \pm 2.206^{\mathrm{b}}$ & $5.94 \pm 2.038^{\mathrm{b}}$ & $-0.837 \pm 2.334$ & $1.754 \pm 1.772^{\mathrm{a}}$ \\
\hline Structure model index (SMI) & $1.105 \pm 0.25$ & $1.782 \pm 0.336^{\mathrm{b}}$ & $1.526 \pm 0.278^{b}$ & $0.812 \pm 0.246$ & $1.267 \pm 0.176^{\mathrm{b}}$ \\
\hline \multicolumn{6}{|l|}{ Cortical parameter } \\
\hline Percent bone volume (\%) (BV/TV) & $92.361 \pm 0.284$ & $91.618 \pm 0.277^{\mathrm{b}}$ & $91.888 \pm 0.282$ & $89.193 \pm 0.255$ & $88.494 \pm 0.29^{\mathrm{b}}$ \\
\hline Bone surface density (1/mm) (BS/TV) & $6.300 \pm 0.68$ & $5.871 \pm 0.077^{\mathrm{a}}$ & $5.967 \pm 0.126$ & $7.218 \pm 0.086$ & $6.999 \pm 0.134^{\mathrm{b}}$ \\
\hline Cortical thickness (mm) (Ct.Th) & $0.522 \pm 0.01$ & $0.487 \pm 0.06^{\mathrm{a}}$ & $0.518 \pm 0.001^{\mathrm{c}}$ & $0.501 \pm 0.007$ & $0.487 \pm 0.008^{\mathrm{b}}$ \\
\hline Cortical area $\left(\mathrm{mm}^{2}\right)($ Ct.Ar $)$ & $32.409 \pm 2.204$ & $29.136 \pm 1.759^{b}$ & $31.623 \pm 2.325^{\mathrm{c}}$ & $23.074 \pm 1.3890$ & $24.392 \pm 2.358$ \\
\hline
\end{tabular}

$\mathrm{NCD}$, normal control diet; $\mathrm{HCD}$, high cholesterol diet; WT, wild-type; $\mathrm{ApoE}^{-{ }^{-}}$, apolipoprotein $\mathrm{E}$ knockout. ${ }^{\mathrm{a}} \mathrm{P}<0.01,{ }^{\mathrm{b}} \mathrm{P}<0.01 \mathrm{vs}$. $\mathrm{NCD}$; ${ }^{\mathrm{c}} \mathrm{P}<0.05$ and ${ }^{\mathrm{d}} \mathrm{P}<0.01$ vs. the corresponding HCD.

have protective effects on bone metabolism in rodents. Obesity reduced bone density in rapidly growing male rats $(25)$. Serum TG levels and body weights were unchanged in the HCD groups compared to those in the controls, indicating that the effects of serum TG levels and weight were excluded in this study. High cholesterol decreased BMD and significantly increased the levels of both bone resorption markers, such as CTX-1 and TRAP, and bone formation markers, such as bone glaprotein (BGP), alkaline phosphatase (ALPL), PINP, indicating that high cholesterol promoted osteoclast-mediated bone resorption and osteoblast-mediated bone formation, resulting in the increased risk of osteoporosis in male rodents. These findings were somewhat inconsistent with those of previous research involving female rodents (26). You et al (26) recently reported that HCD administration did not promote bone resorption but inhibited bone formation in female rats. We attribute the above discrepancies mainly to the difference in the genders of the mice used in the two studies. Other authors aimed to study the effects of an HCD on female OP. Since differences in bone metabolism due to differences in endocrine and paracrine factors exist between the sexes (27-29), the effects of hypercholesterolaemia on male and female bone metabolism and the underlying mechanisms may be different. In addition, the authors of one study used an HCD containing $20 \%$ lard, which induces obesity and high TG, leading to bone formation inhibition (25). Tintut et al (30) recently reported that hypercholesterolaemia promotes osteoclastic differentiation and resorptive activity in vivo, consistent with our study. These findings supported our clinical hypothesis. In our animal study, high cholesterol caused trabecular and cortical microstructural damage and reduced bone mechanical strength. Therefore, we believe that high cholesterol damages bone microstructure and decreases bone mechanical strength by excessively enhancing bone transformation, leading to a higher risk of OP and a higher risk of fracture in males. In addition, our findings indicate that the changes observed in the HCD group can be slightly attenuated by withdrawal of the HCD, suggesting that diet modification can ameliorate the negative effects of hypercholesterolaemia on bone.

The ApoE-KO rat is a useful model for studying the functions of congenital hypercholesterolaemia. Rats lacking ApoE have congenital hypercholesterolaemia, as demonstrated by their elevated serum cholesterol levels and unchanged serum TG levels $(31,32)$. To the best of our knowledge, this study is the first to investigate the effects of congenital hypercholesterolaemia on male bone. In our ApoE-KO male rat model, $\mathrm{ApoE}^{-/-}$rats displayed an enormous increase in their serum TC levels but no change in their serum TG levels or weights compared to those in control rats. We found that the effects of endogenous hypercholesterolaemia on BMD, bone turnover, bone microstructure and bone strength were similar to those of exogenous hypercholesterolaemia. We regarded endogenous hypercholesterolaemia as a positive-control group. Our animal findings suggest that both endogenous and exogenous hypercholesterolaemia promote the development of male OP by exceedingly promoting both osteoclast activity and osteoblast activity.

As the above results show, high cholesterol can boost both osteoclast and osteoblast functions in vivo. To research the direct influence of cholesterol on both osteoclast and osteoblast functions, we designed an in vitro cell experiment. Since many cell studies have shown that cholesterol promotes enhanced osteoclast differentiation and osteoclast activity (33) and the conclusion of the effects of cholesterol on osteoblasts is contradictory, we studied only the effects of cholesterol on osteoblast function. We found that free cholesterol increased the expression levels of genes involved in osteoblastic function and that cholesterol can promote osteoblastic function. Li et al (34) documented that cholesterol-treated mesenchymal stem cells (MSCs) showed increased expression levels of 
A a

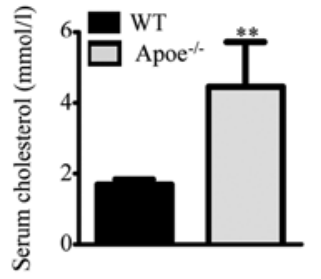

b

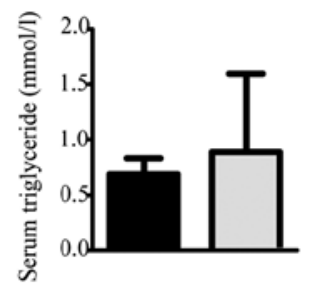

c

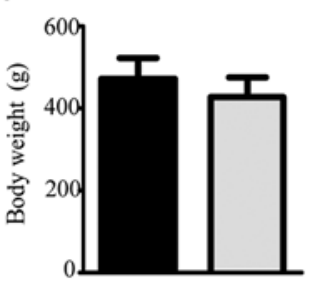

B

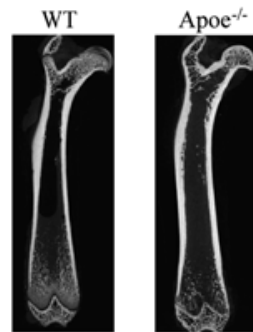

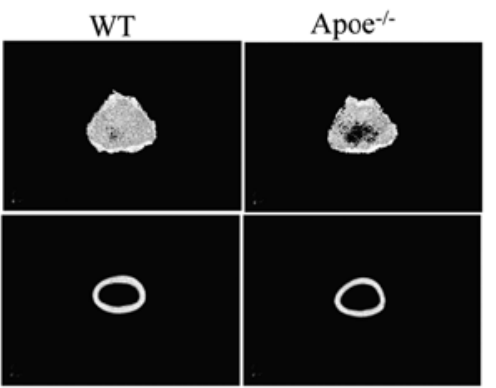

D a

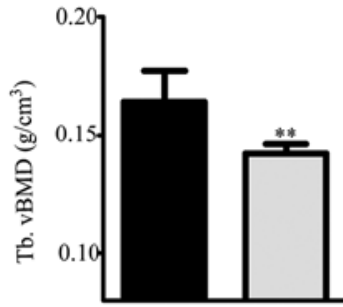

b

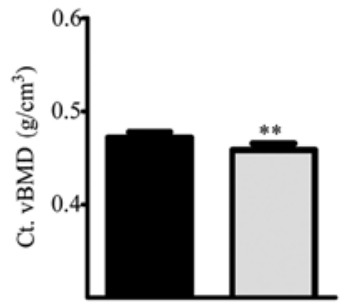

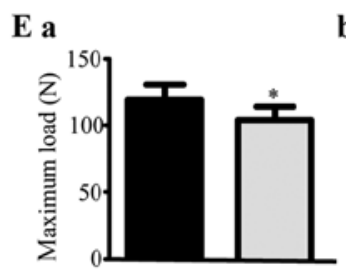
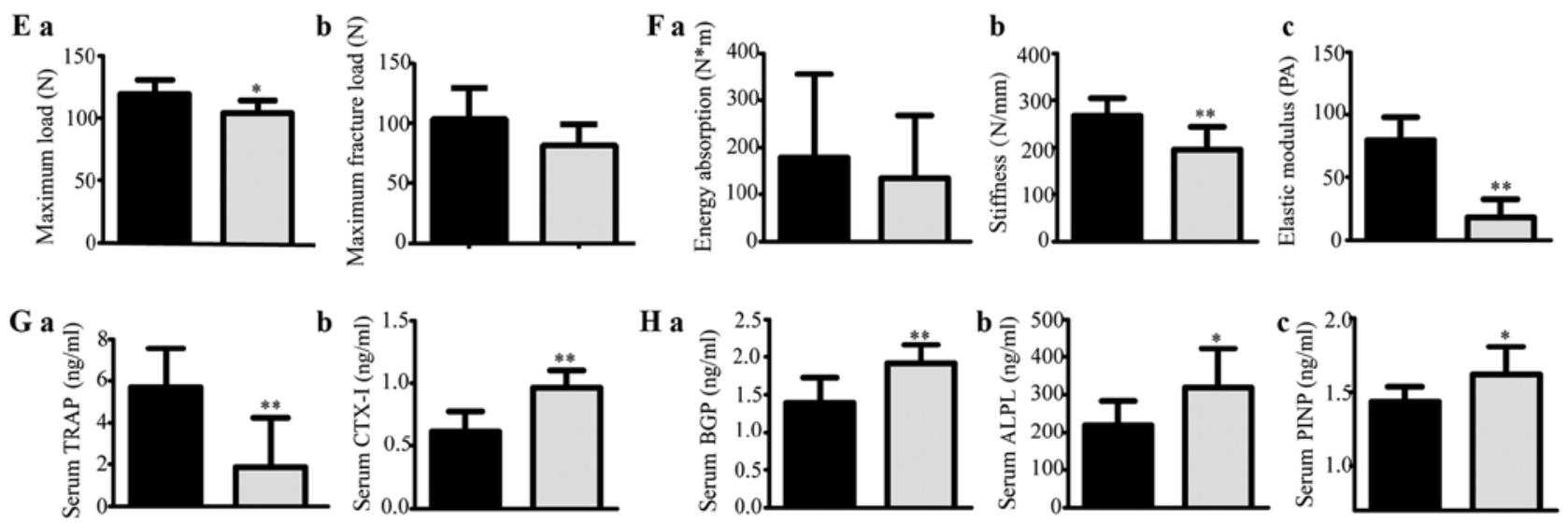

Figure 2. Effects of endogenous hypercholesterolaemia induced by ApoE knockout on male rats. (A) Serum (a) TC and (b) TG levels, and (c) body weight of the rats. (B) Representative 2D mCT images showing the trabecular and cortical microarchitectures of the femurs of each group. (C) Representative 3D mCT images showing the trabecular microarchitecture of the distal femur and the cortical microarchitecture of the midfemoral diaphyses. (D) Trabecular volumetric BMD (Tb. vBMD) (a) and cortical volumetric BMD (Ct. vBMD) (b) of the femur, as analysed via mCT. (E and F) The bone biomechanical parameters of the femoral diaphysis were calculated using a three-point bending test, and the maximum load (E-a), maximum fracture load (E-b), energy absorption (F-a), stiffness (F-b) and elastic modulus (F-c) of the femoral diaphysis were analysed. (G) The levels of the serum bone resorption markers TRAP (a) and beta-CTX (b) in rats were measured by ELISA kits. (H) The levels of the serum bone formation markers BGP (a), ALPL (b) and PINP (c) in rats were measured by ELISA kits. ${ }^{~} \mathrm{P}<0.05$ and ${ }^{* *} \mathrm{P}<0.01$ vs. the corresponding WT group. TC, triglyceride; LDL-C, low-density lipoprotein cholesterol; HDL-C, high-density lipoprotein cholesterol; TG, triglyceride; BMD, bone mineral density; BGP, bone glaprotein; ALPL, alkaline phosphatase; PINP, type I anterior collagen amino terminal peptide; WT, wild-type; ApoE ${ }^{--}$, ApoE-knockout (KO).

osteogenic lineage markers, supportive of our conclusion. Liu et al (35) reported that the proliferation/viability of primary alveolar osteoblast cells (AOBs) was significantly decreased by simvastatin (a cholesterol-lowering drug) treatment at different concentrations, findings consistent with ours. However, You et al (26) reported that free cholesterol inhibits the proliferation and differentiation of osteoblasts in vitro, findings inconsistent with ours. We attribute the above difference to the fact that the free cholesterol treatment doses used in that study were different from those used in this study, as our cholesterol treatment doses were lower. In the present study, we observed substantial death in the osteoblast population treated with cholesterol at a concentration higher than $20 \mu \mathrm{g} / \mathrm{ml}$; thus, we chose a cholesterol treatment dose below $20 \mu \mathrm{g} / \mathrm{ml}$ in subsequent experiments to avoid the toxicity induced by cholesterol levels higher than $20 \mu \mathrm{g} / \mathrm{ml}$. The authors of the other study used the MC3T3-E1 osteoblast line, and we used rat calvaria primary osteoblasts, which we deemed ideal osteoblast model cells. Thus, our in vitro experimental results conform more to those of our in vivo animal experiments than to those of the other study. Thus, we believe that our results are more truthful.

The present study had some limitations. In our in vitro experiments, we did not investigate the effects of cholesterol on osteoclasts since many studies have found that cholesterol promotes osteoclastic differentiation and function, the results of which were consistent with those of our study. However, additional studies regarding this issue are needed, as are accurate evaluations of cell signal transduction in osteoblasts and cell function and signal transduction in osteoclasts.

In summary, we found that hypercholesterolaemia can promote the development of male OP by excessively promoting 
A a

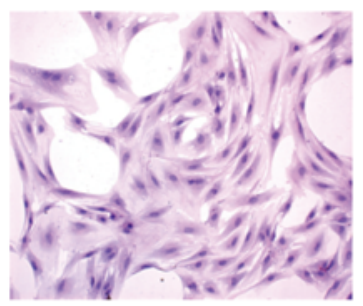

$\mathrm{B}$ a

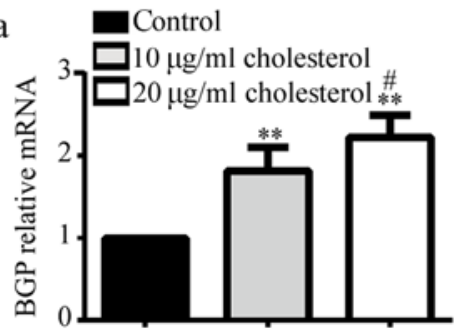

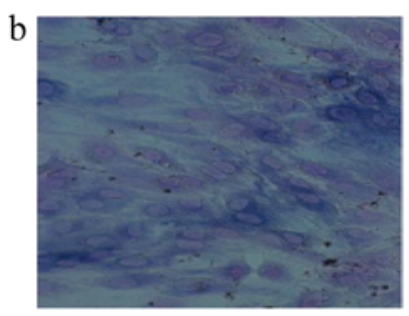

$\mathrm{c}$

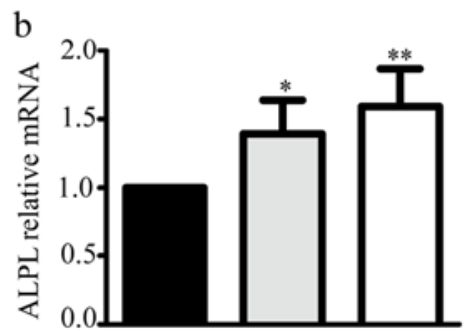

d
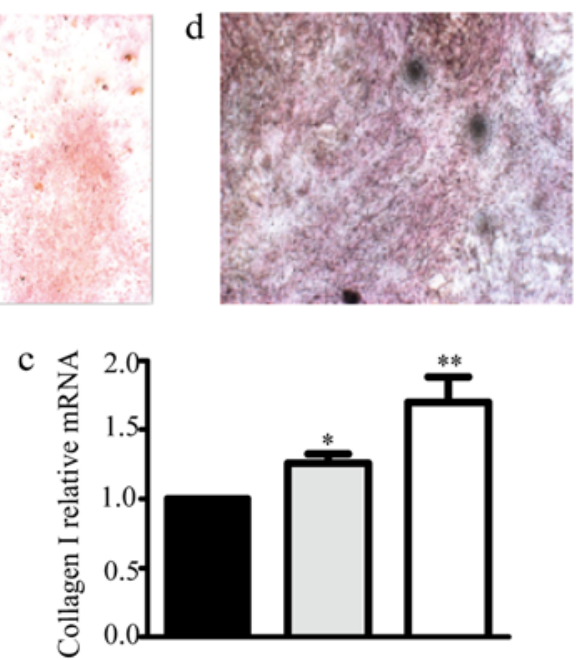

$\mathrm{C}$ a

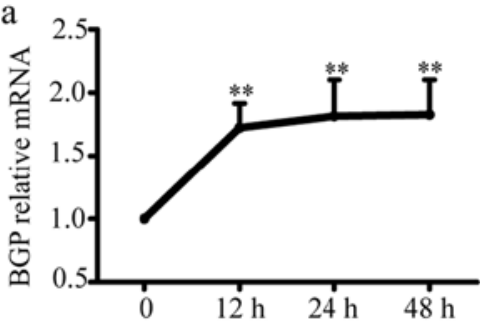

b

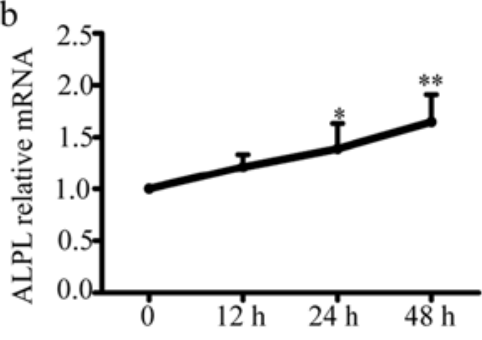

c

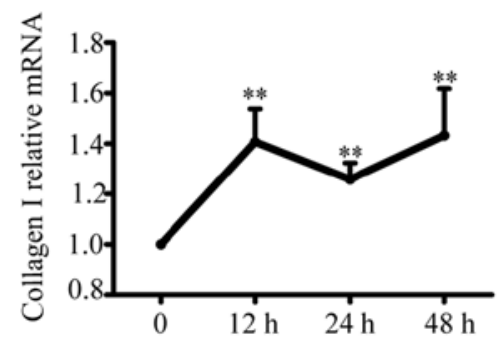

D a

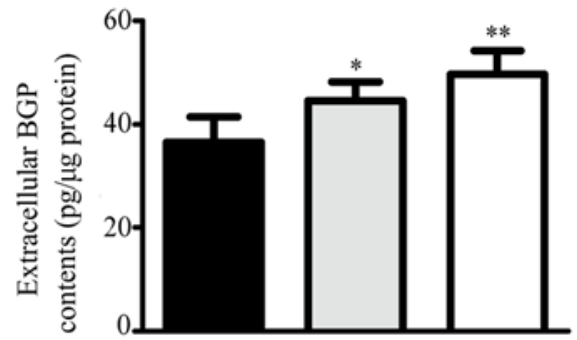

E a

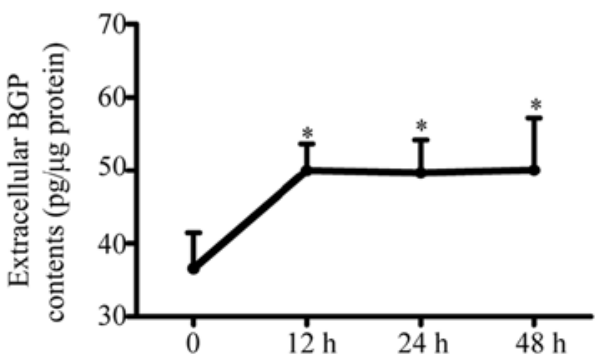

b

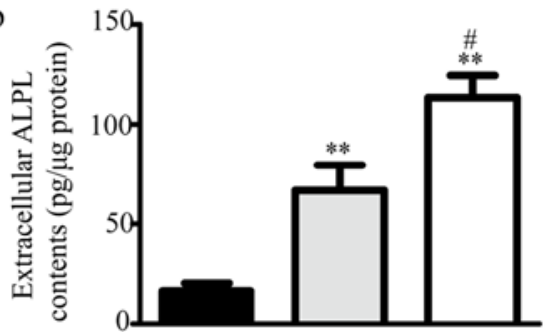

b

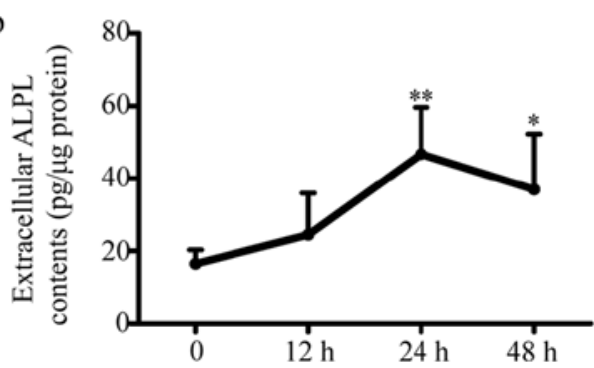

Figure 3. Effects of cholesterol on primary osteoblast function. (A) We evaluated primary osteoblast H\&E staining via microscopy. The cells displayed multiple bumps, via which they appeared to be connected to adjacent cells, as well as large centrally located or shifted cell nuclei (a; x100 magnification). The cytoplasm appeared violet after alkaline phosphatase (ALP) staining ( $b$; 400 magnification). The osteoblasts displayed many round or elliptical opaque nodules, which appeared red after Alizarin red staining (c; x40 magnification) and black after Von Kossa staining, after being cultured for 16 days (d; x40 magnification). (B) BGP (a), ALPL (b) and collagen I (c) mRNA expression levels were determined by reverse transcription-quantitative polymerase chain reaction. " $\mathrm{P}<0.05$, ${ }^{* *} \mathrm{P}<0.01$ vs. control, ${ }^{,} \mathrm{P}<0.05$ vs. $10 \mu \mathrm{g} / \mathrm{ml}$ cholesterol. (C) BGP (a), ALPL (b) and collagen I (c) mRNA expression levels after $10 \mu \mathrm{g} / \mathrm{ml}$ cholesterol treatment for 12,24 and $48 \mathrm{~h} .{ }^{*} \mathrm{P}<0.05,{ }^{* *} \mathrm{P}<0.01$ vs. control. (D) Extracellular BGP (a) and ALPL (b) content was measured by ELISA kits. ${ }^{*} \mathrm{P}<0.05$, ${ }^{* *} \mathrm{P}<0.01$ vs. control, ${ }^{\#} \mathrm{P}<0.05$ vs. $10 \mu \mathrm{g} / \mathrm{ml}$ cholesterol. (E) Extracellular BGP (a) and ALPL (b) content after treatment with $10 \mu \mathrm{g} / \mathrm{ml}$ cholesterol for 12,24 and $48 \mathrm{~h}$. "P<0.05, ${ }^{* *} \mathrm{P}<0.01$ vs. control. BGP, bone glaprotein; ALPL, alkaline phosphatase.

both osteoclast activity and osteoblast activity, which excessively enhances the bone transformation rate in men. Both endogenous and exogenous hypercholesterolaemia in two male animal experimental models were used to validate the clinical findings. Again, we found that hypercholesterolaemia damaged the bone microstructure, thus, increasing the risk of osteopenia or OP and reducing bone strength, resulting in a higher risk of fracture. Many in vitro studies have shown that 
cholesterol enhances osteoclast differentiation and osteoclast activity (34). In vitro, cholesterol directly upregulated the functions of osteoblasts. Based on these results, the detrimental effects of hypercholesterolaemia on bone health probably represent a higher risk of high-turnover osteoporosis and future fractures in men. In conclusion, we wish to emphasize the importance of preventing and treating hypercholesterolemia to prevent osteoporotic bone loss and of monitoring bone metabolic markers, preferably in conjunction with lowering BMD, in men with hypercholesterolemia for the effective prevention of bone loss and subsequent fractures. In addition, our findings provide a theoretical basis for the development of treatments for high cholesterol-induced osteoporosis.

\section{Acknowledgements}

We are very thankful to the medical postgraduates of Shandong Provincial Hospital and the Jinan Health Organization for their cooperation in sample collection, obtaining consent from patients and filling out the voluntary questionnaire. We are very thankful to the Clinical Laboratory Department of Shandong Provincial Hospital for providing the serum indices for the clinical tests.

\section{Funding}

The present study was supported by grants from the Key Research and Development Plan Project of Shandong Province (grant no. 2016 GSF201025), the National Natural Science Foundation of China (grant no. 81370892) and the Shandong Taishan Scholars Specially-invited Expert Plan.

\section{Availability of data and materials}

The datasets obtained and/or analysed in the current study are available from the corresponding author upon reasonable request.

\section{Authors' contributions}

JX conceived the study and participated in its design and coordination; YZ conceived and participated in the design of the study, carried out the animal and cell experiments, participated in the data analysis and drafting, and wrote the manuscript; TD and HoZ participated in the design of the study and carried out some of the cell experiments. HaZ, QG and MZ guided the epidemiological investigation, the sample collection, the questionnaire and serum index tests. CY and SS participated in the design of the study and carried out some animal experiments. All authors read and approved the manuscript and agree to be accountable for all aspects of the research in ensuring that the accuracy or integrity of any part of the work are appropriately investigated and resolved.

\section{Ethics approval and consent to participate}

The study protocol using human subjects was approved by the Ethics Committee of Provincial Hospital Affiliated with Shandong University (Jinan, China) and all participants provided written informed consent before participating in the study. The animal experimental protocol was approved by the Animal Ethics Committee of Shandong Provincial Hospital.

\section{Patient consent for publication}

Not applicable.

\section{Competing interests}

The authors declare that they have no competing interests.

\section{References}

1. Osteoporosis: Review of the evidence for prevention, diagnosis and treatment and cost-effectiveness analysis. Introduction. Osteoporos Int 8 (Suppl 4): S7-S80, 1998.

2. Looker AC, Orwoll ES, Johnston CC Jr, Lindsay RL, Wahner HW, Dunn WL, Calvo MS, Harris TB and Heyse SP: Prevalence of low femoral bone density in older U.S. adults from NHANES III. J Bone Miner Res 12: 1761-1768, 1997.

3. Cummings SR and Melton LJ: Epidemiology and outcomes of osteoporotic fractures. Lancet 359: 1761-1767, 2002.

4. Trivedi DP and Khaw KT: Bone mineral density at the hip predicts mortality in elderly men. Osteoporos Int 12: 259-265, 2001.

5. Wu LY, Yang TC, Kuo SW, Hsiao CF, Hung YJ, Hsieh CH, Tseng HC, Hsieh AT, Chen TW, Chang JB, et al: Correlation between bone mineral density and plasma lipids in Taiwan. Endocr Res 29: 317-325, 2003.

6. Huang Y, Gao L, Xie X and Tan SC: Epidemiology of dyslipidemia in Chinese adults: Meta-analysis of prevalence, awareness, treatment, and control. Popul Health Metr 12: 28, 2014.

7. Zheng G: Relevant Guidelines for the management of abnormal lipids in 2016 and updated interpretation of expert consensus. World Clinical Drugs 7: 441-444, 2017.

8. Turner $\mathrm{CH}$ and Burr DB: Basic biomechanical measurements of bone: A tutorial. Bone 14: 595-608, 1993.

9. Li XF, Zhao JM, Su W, et al: Primary culture and identification of rat osteoblasts. J Clin Rehabil Tissue Eng Res 15: 990-994, 2011.

10. Mooradian AD: Dyslipidemia in type 2 diabetes mellitus. Nat Clin Pract Endocrinol Metab 5: 150-159, 2009.

11. Koshiyama H, Wada Y and Nakamura Y: Hypercholesterolemia as a possible risk factor for osteopenia in type 2 diabetes mellitus. Arch Intern Med 161: 1678-1678, 2001.

12. Zhang N, Zhang H, Zhang X, Zhang B, Wang F, Wang C, Zhao M, Yu C, Gao L, Zhao J and Guan Q: The relationship between endogenous testosterone and lipid profile in middle-aged and elderly Chinese men. Eur J Endocrinol 170: 487-494, 2014.

13. Wongdee $\mathrm{K}$ and Charoenphandhu $\mathrm{N}$ : Osteoporosis in diabetes mellitus: Possible cellular and molecular mechanisms. World J Diabetes 2: 41-48, 2011.

14. Gaffney CD, Pagano MJ, Kuker AP, Stember DS and Stahl PJ: Osteoporosis and low bone mineral density in men with testosterone deficiency syndrome. Sex Med Rev 3: 298-315, 2015.

15. Saad F, Röhrig G, von Haehling S and Traish A: Testosterone deficiency and testosterone treatment in older men. Gerontology 63: 144-156, 2017.

16. Tsourdi E, Rijntjes E, Köhrle J, Hofbauer LC and Rauner M: Hyperthyroidism and Hypothyroidism in male mice and their effects on bone mass, bone turnover, and the Wnt inhibitors sclerostin and Dickkopf-1. Endocrinology 156: 3517-3527, 2015.

17. Reid IR: Relationships between fat and bone. Osteoporos Int 19: 595-606, 2008.

18. Murdolo G, Bartolini D, Tortoioli C, Piroddi M, Iuliano L and Galli F: Lipokines and oxysterols: Novel adipose-derived lipid hormones linking adipose dysfunction and insulin resistance. Free Radic Biol Med 65: 811-820, 2013.

19. Verma S, Rajaratnam JH, Denton J, Hoyland JA and Byers RJ: Adipocytic proportion of bone marrow is inversely related to bone formation in osteoporosis. J Clin Pathol 55: 693-698, 2002.

20. Edwards CJ, Hart DJ and Spector TD: Oral statins and increased bone-mineral density in postmenopausal women. Lancet 355: 2218-2219, 2000 . 
21. Meier CR, Schlienger RG, Kraenzlin ME, Schlegel B and Jick H: HMG-CoA reductase inhibitors and the risk of fractures. JAMA 283: 3205-3210, 2000.

22. Majima T, Shimatsu A, Komatsu Y, Satoh N,Fukao A, Ninomiya K, Matsumura T and Nakao K: Increased bone turnover in patients with hypercholesterolemia. Endocr J 55: 143-151, 2008.

23. Halade GV, Rahman MM, Williams PJ and Fernandes G: High fat diet-induced animal model of age-associated obesity and osteoporosis. J Nutr Biochem 21: 1162-1169, 2010.

24. Tatsumi S, Ito M, Asaba Y, Tsutsumi K and Ikeda K: Life-long caloric restriction reveals biphasic and dimorphic effects on bone metabolism in rodents. Endocrinology 149: 634-641, 2008.

25. Chen JR, Lazarenko OP, Wu X, Tong Y, Blackburn ML, Shankar K, Badger TM and Ronis MJ: Obesity reduces bone density associated with activation of PPAR $\gamma$ and suppression of Wnt $/ \beta$-catenin in rapidly growing male rats. PLoS One 5: e13704, 2010.

26. You L, Sheng ZY, Tang CL, Chen L, Pan L and Chen JY: High cholesterol diet increases osteoporosis risk via inhibiting bone formation in rats. Acta Pharmacol Sin 32: 1498-1504, 2011.

27. Yan L, Prentice A, Zhou B, Zhang H, Wang X, Stirling DM, Laidlaw A, Han Y and Laskey A: Age- and gender-related differences in bone mineral status and biochemical markers of bone metabolism in Northern Chinese men and women. Bone 30 412-415, 2002

28. Khosla S, Melton LJ III and Riggs BL: Osteoporosis: Gender differences and similarities. Lupus 8: 393-396, 1999.

29. Ebbesen EN, Thomsen JS, Beck-Nielsen H,Nepper-Rasmussen HJ and Mosekilde L: Age- and gender-related differences in vertebral bone mass, density, and strength. J Bone Miner Res 14: 1394-1403, 1999.
30. Tintut Y, Morony S and Demer LL: Hyperlipidemia promotes osteoclastic potential of bone marrow cells ex vivo. Arterioscler Thromb Vasc Biol 24: e6-e10, 2004.

31. Wei S, Zhang Y, Su L, He K, Wang Q, Zhang Y, Yang D, Yang Y and Ma S: Apolipoprotein E-deficient rats develop atherosclerotic plaques in partially ligated carotid arteries. Atherosclerosis 243: 589-592, 2015.

32. Ekuni D, Yoneda T, Endo Y, Kasuyama K, Irie K, Mizutani S, Azuma T, Tomofuji T and Morita M: Occlusal disharmony accelerates the initiation of atherosclerosis in apoE knockout rats. Lipids Health Dis 13: 144, 2014.

33. Luegmayr E, Glantschnig H, Wesolowski GA, Gentile MA, Fisher JE, Rodan GA and Reszka AA: Osteoclast formation, survival and morphology are highly dependent on exogenous cholesterol/lipoproteins.CellDeathDiffer 11 (Suppl1):S108-S118, 2004.

34. Li H, Guo $\mathrm{H}$ and $\mathrm{Li} \mathrm{H}$ : Cholesterol loading affects osteoblastic differentiation in mouse mesenchymal stem cells. Steroids 78: 426-433, 2013.

35. Liu S, Bertl K, Sun H, Liu ZH, Andrukhov O and Rausch-Fan X: Effect of simvastatin on the osteogenetic behavior of alveolar osteoblasts and periodontal ligament cells. Hum Cell 25: 29-35, 2012.

This work is licensed under a Creative Commons Attribution-NonCommercial-NoDerivatives 4.0 International (CC BY-NC-ND 4.0) License. 